EMISSION OF COMPLEX FRAGMENTS IN COMPOUND NUCLEUS DECAY

\section{L.G. MORETTO}

Nuclear Science Division Lawrence Berkeley Laboratory University of California Berkeley, California 94720

\author{
LBL -21380
}

DE86 012501

International Symposium on Nuclear Fission and Heavy Ion Induced Reactions, Rochester. New York, April 20-22, 1986

This work was supported by the Director, Office of Energy Research, Division of Nuclear Physics of the Office of High Energy and Nuclear Physics of the U.S. Department of Energy under Contract DE-ACO3-76SF00098. 
EMISSION OF COMPLEX FRAGMENTS IN COMPOUND NUCLEUS DECAY

L.G. MORETTO

Nuclear Science Division, Lawrence Berkeley Laboratory, University of California, Berkeley, California 94720

Abstract The compound nucleus mechanism for complex fragment emission is discussed theoretically and demonstrated experimentally. The role of the potential energy as a function of mass asymmetry is shown in experimental charge distributions. This process is followed from near the threshold up to bombarding energies of several tens of MeV A.

Complex particles have appeared very early and obscurely in the game of nuclear physics, ever since radiochemists bombardef a variety of targets with high energy protons and managed to fish out of the resulting soup a few light radioactive elements. 1 The same particles made their debut in the society of instrumental nuclear physics when they were detected by means of particle telescopes in the reaction of $U, A g$ with $\mathrm{GeV}$ protons, ${ }^{2}$ although nobody paid much attention to them either. Recently a new interest, probably stirred by the abundance of their production in heavy ion reactions, has brought these particles in the limelight. At least two interpretations have been advanced regarding their origin. The first claims these particles to be the result of nuclear shattering or cold fragmentation. 3 The second sees them produced as droplets condensing out of a vapor at or near the critical temperature. ${ }^{4}$ We felt it would be worth trying to clarify the picture by going way down in energy and in particular by checking whether the compound nucleus itself could not be one of their possible sources through a mechanism somehow intermediate between standard evaporation and fission.

In fact the experimental distinction between the processes of evaporation and fission in relatively heavy compound nuclei can be understood in terms of a specific topological feature in the liquid drop model potential 


\section{L.G. MORETTO}

energy surface $V(Z)$ as a function of mass asymmetry $Z$. This feature is a deep minimum at symmetry (fission region) flanked at greater asymmetries by the Businaro-Gallone mountains, which in turn descend at even larger asymmetries ("evaporation" region). The corresponding mass distribution expected for compound nucleus decay is approximately proportional to $\exp \left[-V(Z) / T_{Z}\right]$, where $T_{2}$ is the temperature of the conditional saddle point, and indeed shows a peak at symetry (fission peak) and two wings at the extreme asymmetries (evaporation wings).

In progressively lighter compound nuclei, the potential energy surface undergoes a topological change as the fissionability parameter $x$ crosses the Businaro-Gallone point. At this point the second derivative of the potential energy with respect to the mass asymmetry coordinate evaluated at symmetry vanishes. Thus, below the BusinaroGallone point. there is no longer a true fission saddle

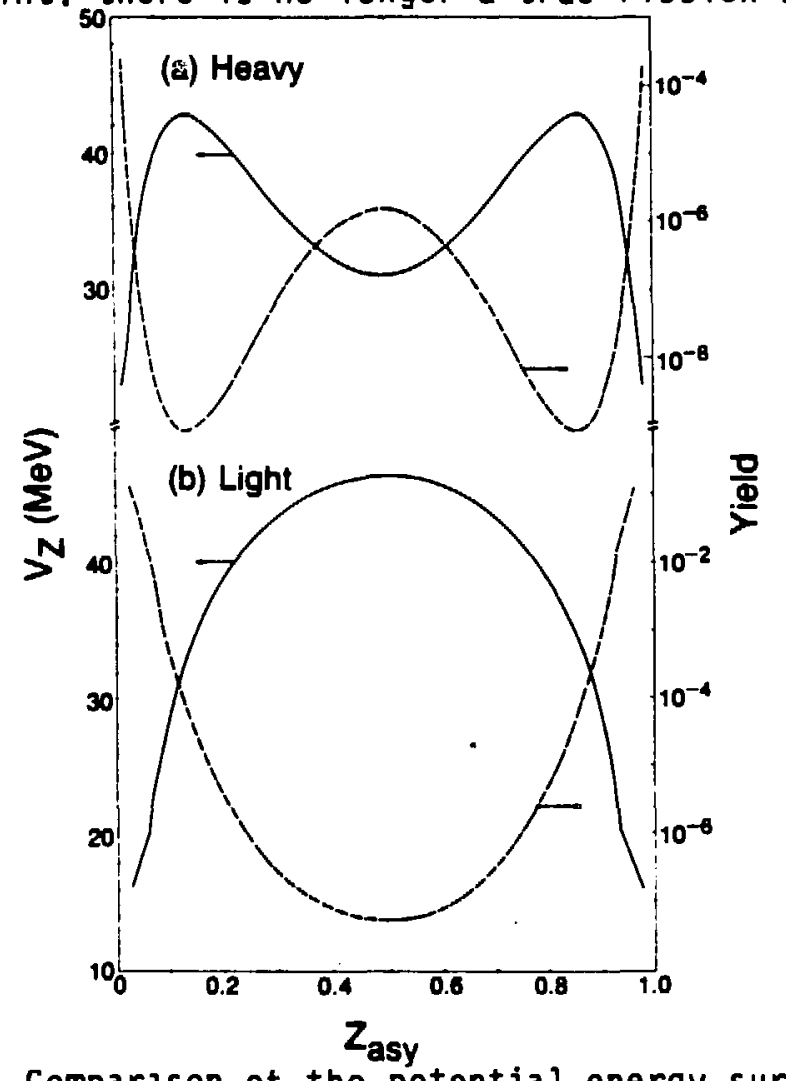

FIGURE 1 Comparison of the potential energy surfaces (solid curve) and expected yields (dashed curve) for a) a heavy $C N\left(A u\right.$ at $\ell=0$ and $E^{\star}=97 \mathrm{MeV}$ ) and b) a light $C N$ ( $G e$ at $\ell=0$ and $E^{\star}=72 \mathrm{MeV}$ ). 
point, and the monotonically increasing potential energy towards symmetry determines the disappearance of fission as a process in any way distinct from evaporation. In other words the mass distribution should show the two evaporation wings extending as far as symmetry where a minimum should be observed. These features are illustrated in Figure 1.

Within this framework, we have developed a theory that describes, in a continuous way, the transition from light particle emission to fission. 5 This theory also predicts changes in the shapes of both the kinetic energy spectra and angular distributions of the emitted fragments as their masses increase from a-particles toward fission fragments. These predictions ${ }^{5}$ are amenable to experimental test; unfortunately, there is an almost complete lack of experimental data to be compared with the above unified treatments or with more standard formalisms.

We obtained the first experimental evidence for the emission of complex nuclei from helium through fluorine by compound nuclei produced in the reaction $90 \mathrm{MeV}{ }^{3} \mathrm{He}+$ nat Ag. ${ }^{6}$ The specific choice of ${ }^{3} \mathrm{He}$ as projectile was dictated by two reasons. On one hand one would like to have a relatively low velocity projectile in order to minimize pre-equilibrium losses, but massive enough to bring in sufficient energy. On the other, the mass of the projectile should be sufficiently smaller than those of the complex fragments of interest in order to rule out the ambiguity of projectile fragmentation or multinucleon transfer.

In order to determine the existence of an isotropically emitting source and its velocity, the laboratory energy spectra were transformed into invariant cross-section plots in velocity space which are presented in Figure 2 . The peak cross section for a heavy complex fragment, such as carbon, has a constant value and occurs at the same c.m. velocity from $170^{\circ}$ to $40^{\circ}$ (as indicated by the position of the $X$ 's relative to the circular arc). At the most forward angle the peak cross section occurs at a slightly increased velocity. Similarly, the higher velocity region (the region near the arc with the larger radius) shows no significant change in the backward hemisphere, but does stretch out at forward angles. For a light complex fragment such as $L i$. the peak of the cross section occurs at a constant c.m. velocity for a smaller backward angle region $\left(170^{\circ}\right.$ to $\left.120^{\circ}\right)$. Forward of $120^{\circ}$ the peak increases both in cross section and in velocity. The slope of the high-energy tail does not change significantly for the three most backward angles, but the intensity of the tail increases as the scattering angle decreases. The ${ }^{9} \mathrm{Be}$ and $\mathrm{B}$ fragments 


\section{L.G. MORETTO}

$70 \mathrm{MeV} \quad{ }^{3} \mathrm{He}+{ }^{\text {nal }} \mathrm{Ag}$

a) $\mathrm{Li}$

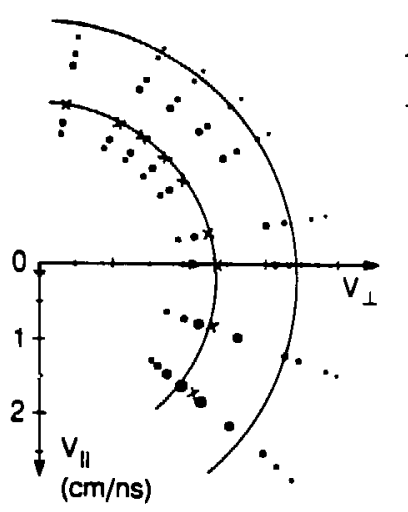

c) Boron

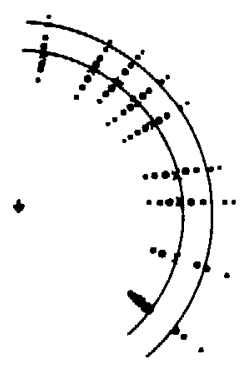

b) ${ }^{9} \mathrm{Be}$

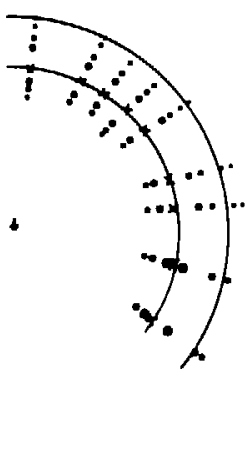

d) Carbon

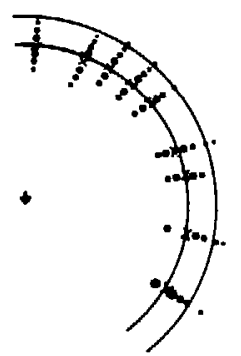

FIGURE 2 Invariant cross section plots

$\left(\alpha \frac{1}{v^{2}} \frac{d^{2} \sigma}{d \Omega d V}\right)$ for representative ejectiles (Li, ${ }^{9} B e$,

$B$, and $C$ ). The diameter of the dots is proportional to the logarithm of the cross section and the $X ' s$ indicate the peak of velocity distribution. The two large arcs are sections of circles centered on the c.m. velocity (center arrow) appropriate for complete fusion. The beam direction $\left(0^{\circ}\right)$ is indicated by the c.m. velocity vector.

show a behavior intermediate between that of $L i$ and $C$. In general, the heavier ejectiles show patterns more consistent with the emission from a single source.

Two conclusions can be drawn from these invariant crosssection plots. First, for all elements there is an angular region in the backward hemisphere where only a single component is observed, which can be characterized as com- 
EMISSION OF COMPLEX FRAGMENTS IN COMPOUND NUCLEUS DECAY

pound nucleus emission. This angular region increases and extends to more forward angles as the ejectile mass

increases. Second, there is a component of non-compound nucleus emission that results in harder energy (or velocity) spectra at forward angles.

The mean energies of the spectra are Coulomb-like and increase as the charge of the fragment increases. The most interesting feature in the energy spectra of the equilibrium component is the evolution from a Maxwellian shape for a-particles or $L i$ ions- through a more symetric shape for $B$ or $C$ to a symmetric shape for the heaviest ejectiles : as predicted in Ref. 5 .

The experimental yields of the equilibrium component are shown in Figure 3 . In order to minimize contributions from sources other than the compound nucleus, we have plotted the yields only for the most backward angle $\left(171^{\circ}\right)$. These yields drop precipitously in going from $z=2$ to $z=$ 3, after which they decrease more slowly. The one exception is the enhanced $z=6$ yield.

The yield from an equilibrium statistical emission process should be roughly proportional to a factor $\exp \left[-B_{2} / T_{Z}\right]$, where $B_{2}$ is the emission barrier for fragment $Z$ and $T_{2}$ is the temperature at the barrier. More quantitatively, the decay width is given by

$$
\begin{aligned}
& r_{z} \propto T_{z}\left[E /\left(E-B_{z}\right)\right]^{2} \exp \left[2 \sqrt{a\left(E-B_{z}\right)}-2 \sqrt{a E}\right] \\
& \tilde{\alpha} \exp \left[-B_{z} / T_{z}\right] .
\end{aligned}
$$

To calculate the theoretical yields, the following expression for the barrier was used

$$
B_{z}=u_{1}+u_{2}+\frac{z_{1} z_{2} e^{2}}{d}+u_{\text {prox }}-u_{C N} \text {, }
$$

where $U_{1}$ is the experimental mass of the light fragment, $\mathrm{U}_{2}, \mathrm{U}_{\mathrm{CN}}$ are the droplet model masses of the residual and compound nucleus, respectively, and $U_{\text {prox }}$ is the proximity potential. The center-to-center distance $d$ in the interfragment Coulomb term was taken to be $d=1.225\left(A_{j}^{1 / 3}+\right.$ $\left.A_{2}^{l / 3}\right)+2 \mathrm{fm}$. The addition of $2 \mathrm{fm}$ was done to obtain rough agreement with the energy spectra. The temperature ( $T_{2}$ ) was evaluated using $E-B=a T^{2}$. A compound nucleus excitation energy (E) of $102 \mathrm{MeV}$ (the value for full momentum transfer) and a level density parameter (a) of $A / B$ were assumed. The calculated yields (Eq. 1) for each iso- 
L.G. MORETTO

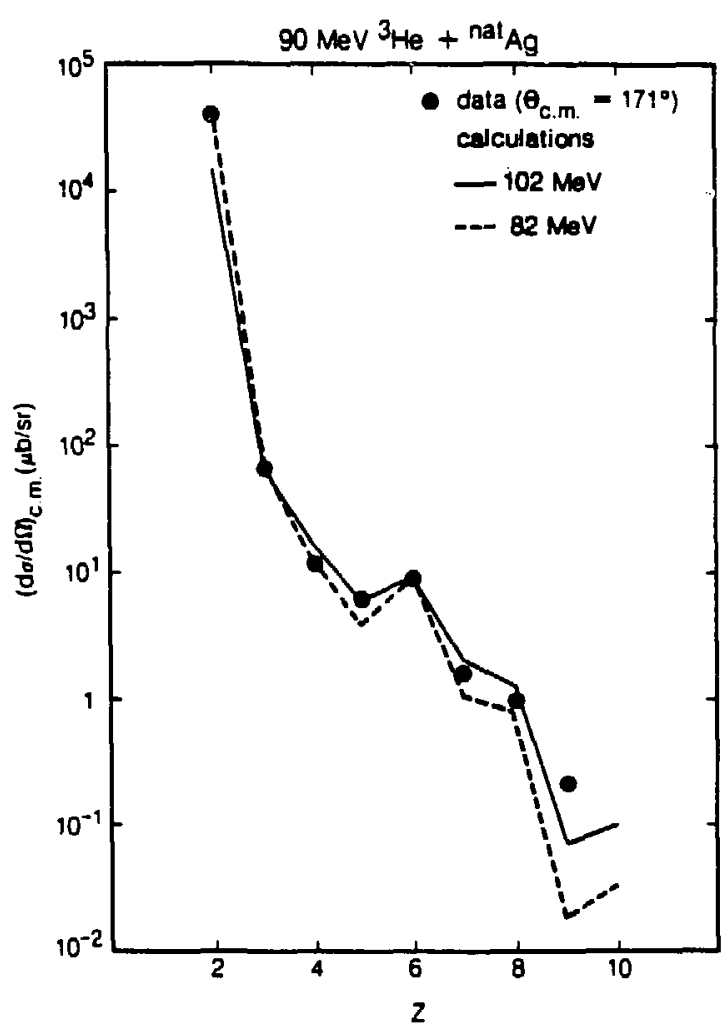

FIGURE 3 Experimental (circles) and theoretical yields versus ejectile atomic number $(Z)$.

tope were multiplied by $2 I+1$ (where $I$ is the ground state spin of the light fragment) and then summed. The theoretical ejectile yields were calculated as a ratio $\Gamma_{2} / r_{6}$ and have been normalized to the data at $Z=6$ in Figure 3 .

The agreement between the data (circles) and this simple equilibrium statistical calculation (solid line) is exceptionally good for $z=3-9$. The calculation underpredicts the a-particle yield because it only takes into account first chance emission, whereas substantial amounts of higher chance a-emission occur. Precompound emission is expected to leave the compound nucleus with a broad excitation energy distribution with a most probable value of $\sim 85 \mathrm{MeV}$. A calculation (not shown) with this lower excitation energy also reproduces the relative yieids of the heavy fragments quite well but overpredicts the yield of first chance a-emission. More detailed comparisons between the data and theory require calculations that 
include precompound emission; however, the substantial agreement depicted in Figure 3 does indicate that an equilibrated process is responsible for the emission of these complex fragments.

Complete excitation functions obtained from the $3_{\mathrm{He}}+$ nat $_{A g}$ measurements are shown in Figure 4 for a series of decay products. The measurements were restricted to the backward angles $\left(120^{\circ}-160^{\circ}\right)$ in order to insure measurement of only the equilibrium component.

with increasing bombarding energy, the cross sections (see Figure 4) rise rapidly and then flatten at higher energies. This is a characteristic signature of compound nucleus emission, and reinforces the assignment of compound nucleus decay that was made previously on the basis of data obtained at $90 \mathrm{MeV}$. The cross section for $Z=3$ is a factor of 1000 lower than that for $Z=2$, and for the heavier fragments it is even lower. In spite of these low cross sections, we were able to measure an excitation function over 2-3 orders of magnitude up to $Z=11$, with a detection limit of about $50 \mathrm{nb}$.

The experimental excitation function data have been fitted using a transition state formalism, analogous to that used to fit fission excitation functions.7 As shown in Ref. 5, the decay width for first-chance emission of a fragment of charge $Z$ can be written as

$$
r_{z}=\frac{1}{2 \pi \rho(E)} \int_{0}^{E-B_{z}} \rho_{z}^{*}\left(E-B_{z}-\varepsilon\right) d \varepsilon
$$

where $\rho(E)$ is the compound nucleus level density, $B_{Z}$ is the conditional barrier height, and $\rho_{z}^{\star}\left(E-B_{z}-\varepsilon\right)$ is the level density at the conditional saddle with a kinetic energy $\varepsilon$ in the decay mode. The neutron width $r_{n}$ can be written as

$$
\Gamma_{n}=\frac{2 m R^{2} g}{2 \pi \rho(E)} \int_{0}^{E-B_{n}} c \rho\left(E-B_{n}-\varepsilon\right) d \varepsilon
$$

We make the assumption that the ratio of the decay widths, $r_{z} / r_{n}$, is proportional to the ratio of the cross section for complex fragment emission, $\sigma_{Z}$, to that for complete fusion, of, i.e..

$$
\Gamma_{z} / \Gamma_{n} \simeq \frac{\Gamma_{z}}{\Gamma_{t}} \simeq \frac{\sigma_{z}}{\sigma_{t}} \simeq \frac{\sigma_{z}}{\sigma_{R}}
$$




\section{L.G. MORETTO}

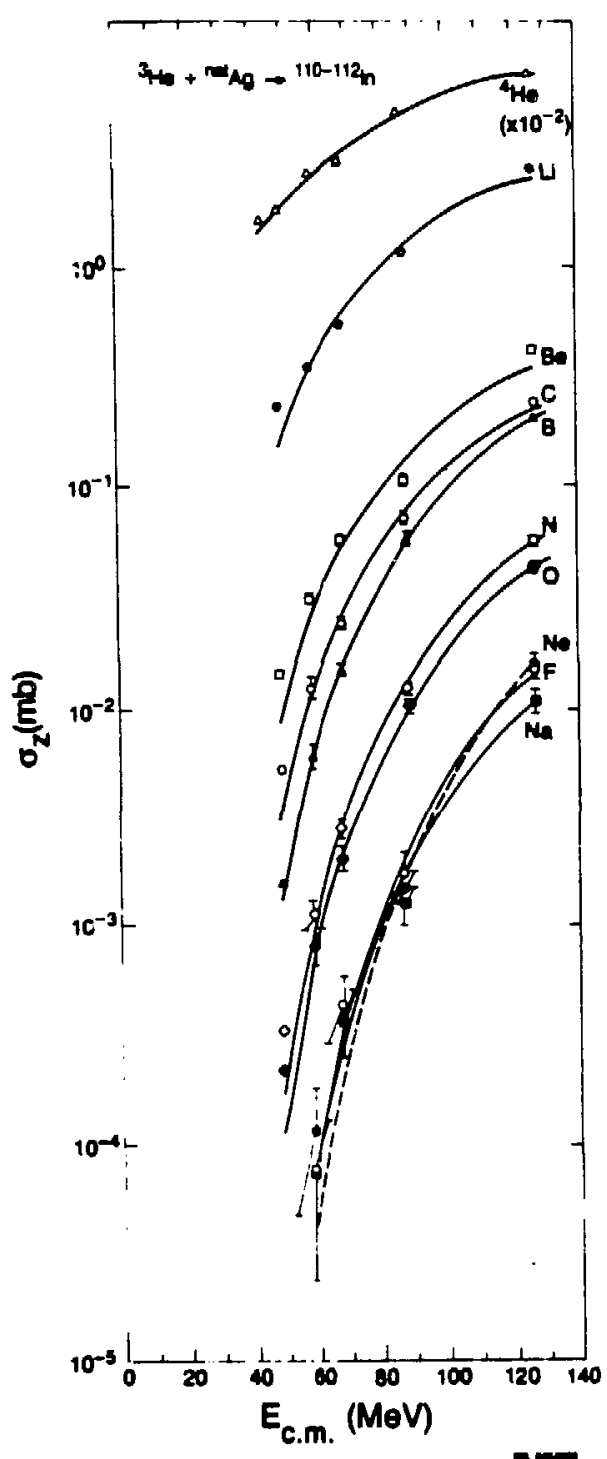

FIGURE 4 Dependence of the total integrated cross sections for emission of complex fragments on the center-of-mass energy, $E_{c . m}$. in the reaction ${ }^{3} \mathrm{He}+$ nat $_{A g}$. The points and error bars correspond to the experimental cross sections. The curves are fits with the parameters of Figure 5 . 
EMISSION OF COMPLEX FRAGMENTS IN COMPOUND NUCLEUS DECAY

This is reasonable in this mass region because $\Gamma_{n} \gg \sum_{z \geq 1} \Gamma_{z}$. One can then calculate $\Gamma_{z} / \Gamma_{n}(E)$

using an appropriate choice for the level density expression. A Fermi gas level density was used because it gives an analytical expression for $r_{z} / \Gamma_{n}$. A simple angular momentum dependence has been included by adding to the barriers the rotational energies appropriate to the ground and saddle point deformations.

Using the above expression for $\Gamma_{7} / \Gamma_{n}$, the barriers $B_{z}$, and the ratio $a_{z} / a_{n}$, of the level density parameters were extracted from fits to the experimental data $\sigma_{Z} / \sigma_{R}$. These fits are shown by the solid lines in Figure 4 . The agreement between the data and the fits is remarkably good for all Z-values and confirms that these products originate from compound nuclear decay.

The barriers extracted from the fits are shown by the circles in Figure 5 as a function of $Z$. The extracted barriers increase dramatically as the exit channel becomes more symmetric. Some evidence of shell effects in the exit channel is visible in the barrier for carbion emission, $Z=6$, which is lower than those of the neighboring elements.

The barriers so obtained can be used to test modern corrections to the liquid drop model, like surface diffuseness and finite range, which become important for strongly indented saddle configurations like those presiding to the emission of complex fragments. A comparison of the standard liquid drop model prediction and of the model incorporating the corrections mentioned above ${ }^{8}$ with our data is also shown in Figure 5 . Clearly our data strongly support the introduction of surface diffuseness and finite range. It is also easy to understand how these and similar data may be very valuable in fixing the relevant parameters of the model.

As mentioned before, the sharp distinction between evaporation and fission in relatively heavy compound nuclei is a result of a specific topological feature of the liquid drop model potential energy surface $V(Z)$ as a function of mass asymetry $z$. The potential energy shows a deep minimum at summetry (fission region) surrounded by the BusinaroGallone nisuntains which in turn descend at even larger asymetries ("evaporation" region). The corresponding mass distribution from compound nucleus decay shows a peak at symmetry (fission peak) and two wings at the extreme asymmetries (evaporation wings). The qualitative dependence of the potential energy and of the mass yield vs. asymmetry is shown in figure la for a heavy nucleus.

With decreasing total mass the potential energy surface 
L.G. MORETTO

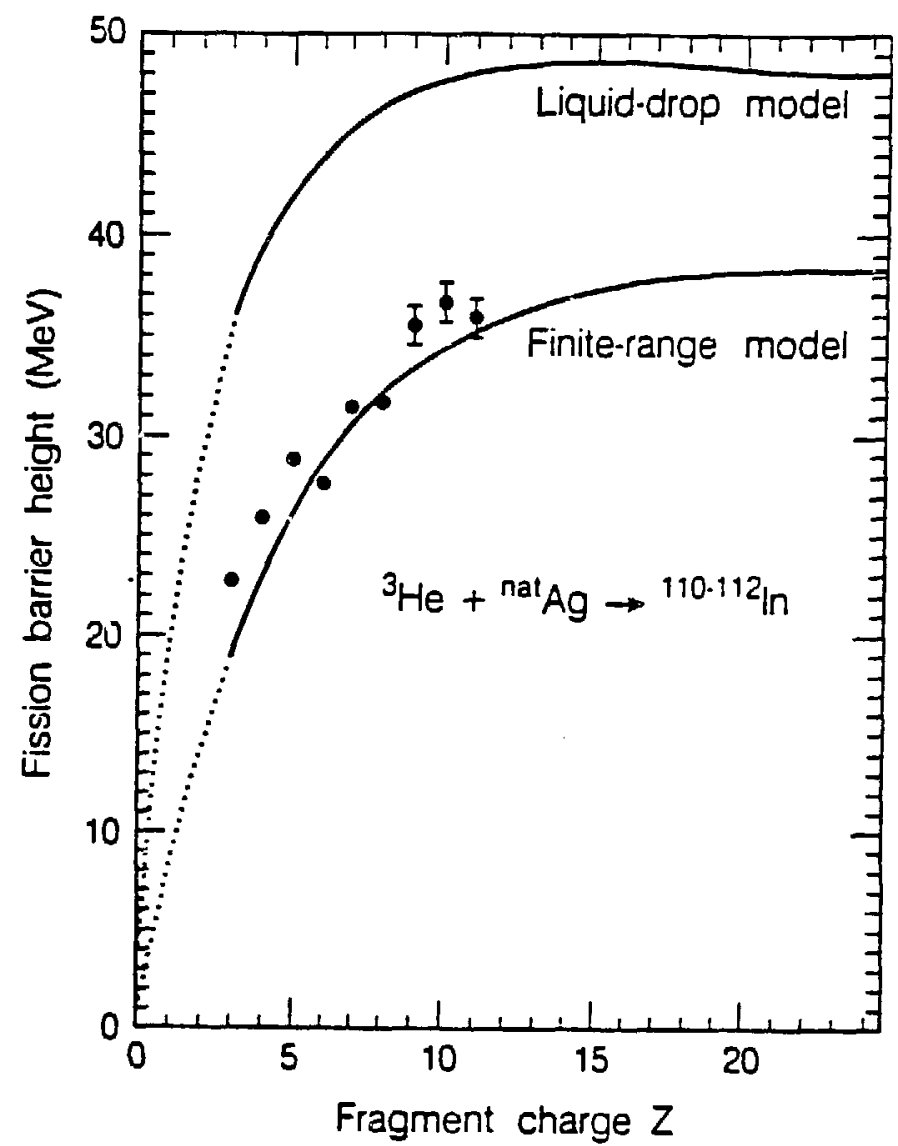

FIGURE 5 The emission barriers, $B_{z}$, extracted in fitting the excitation mission of complex fragments functions as a function of fragment charge. The liquid drop model and finite range model calculations are from ref. 9.

undergoes a qualitative change when the fissility parameter $x$ crosses the so-called Businaro-Gallone point. At this point ( ${ }_{B} B=0.396$ for $\ell=0$ and decreasing for larger $\ell$ values) the second derivative of the potential energy with respect to the mass asymmetry coordinate evaluated at symmetry vanishes. Thus below the Businaro-Gallone point there is no longer a traditional fission saddle point, and fission disappears as a process distinct from evaporation. Thus the mass distribution should show the two evaporation wings extending as far as symmetry where a minimum should be observed. This is illustrated in Figure lb. 


\section{EMISSION OF COMPLEX FRAGMENTS IN COMPOUND NUCLEUS DECAY}

Such a transition has never been observed, as it requires the measurement of the entire mass distribution from symmetry to the extreme asymmetry of $a, p$ evaporation for a series of systems straddling the Businaro-Gallone point. This measurement is made very difficult by the low yield for symmetric decay of the compound nucleus in this general mass region, and by the need to verisy that the fragments were produced by a compound nuclelis mechanism. 6

We have measured ${ }^{9}$ complete charge distributions from protons to symmetric splitting for a variety of nuclei and we have observed the Businaro-Gallone transition. Such a transition is inferred from the disappearance of the fission peak in the mass yie?d as the compound nucleus mass was decreased from $148 \mathrm{Eu}, 102 \mathrm{Rh}$ to $83 \mathrm{Kr}$.

The use of reverse kinematics (projectile heavier than the target) was crucial in performing these measurements. This technique virtually eliminates the problems associated with low cross section measuremerits due to the presence of light element target contaminants. Furthermore, reverse kinematics provides a large center-of-mass (c.m.) velocity which facilitates the verification of full momentum transfer and allows for easy identification of the fragment's atomic number at the higher lab energies. Finally the high energy solution at forward angles corresponds to very backward angles in ordinary kinematics. This enhances the observation of compound nucleus decay and virtually eliminates any possible deep-inelastic contamination.

The velocities of the emitting source and the energies in the source frame of reference for the $93 \mathrm{Nb}+12 \mathrm{C}$ system are shown in Figure 6 . The upper part of this figure demonstrates that, with two extreme mass assumptions, all of the measured products result from the decay of a system with full momentum transfer. For the other systems studied, the extracted source velocities are also independent of $Z$ within a few percent of the velocity expected for full momentum transfer. The deduced $c . m$. energies are shown in the lower portion of figure 6 . These energies are reproduced by a coulomb calculation for two spheres with a surface separation of $2 \mathrm{fm}$. This same separation also reproduces the $c . m$. energies from the $74 \mathrm{Ge}$ induced reactions; however a larger separation is required for the 139 La data. Both the full momentum transfer and the invariance with angle of the c.m. energies seen above are consistent with compound nucleus decay.

The experimental cross sections for $530-\mathrm{MeV} 74 \mathrm{Ge}$, $782-\mathrm{HeV}{ }^{93} \mathrm{Nb}$ and $1157-\mathrm{HeV} 139 \mathrm{La}+9_{\mathrm{Be}}$ systems are shown in Figure 7 . The cross sections are plot+ed as a 
L.G. MORETTO

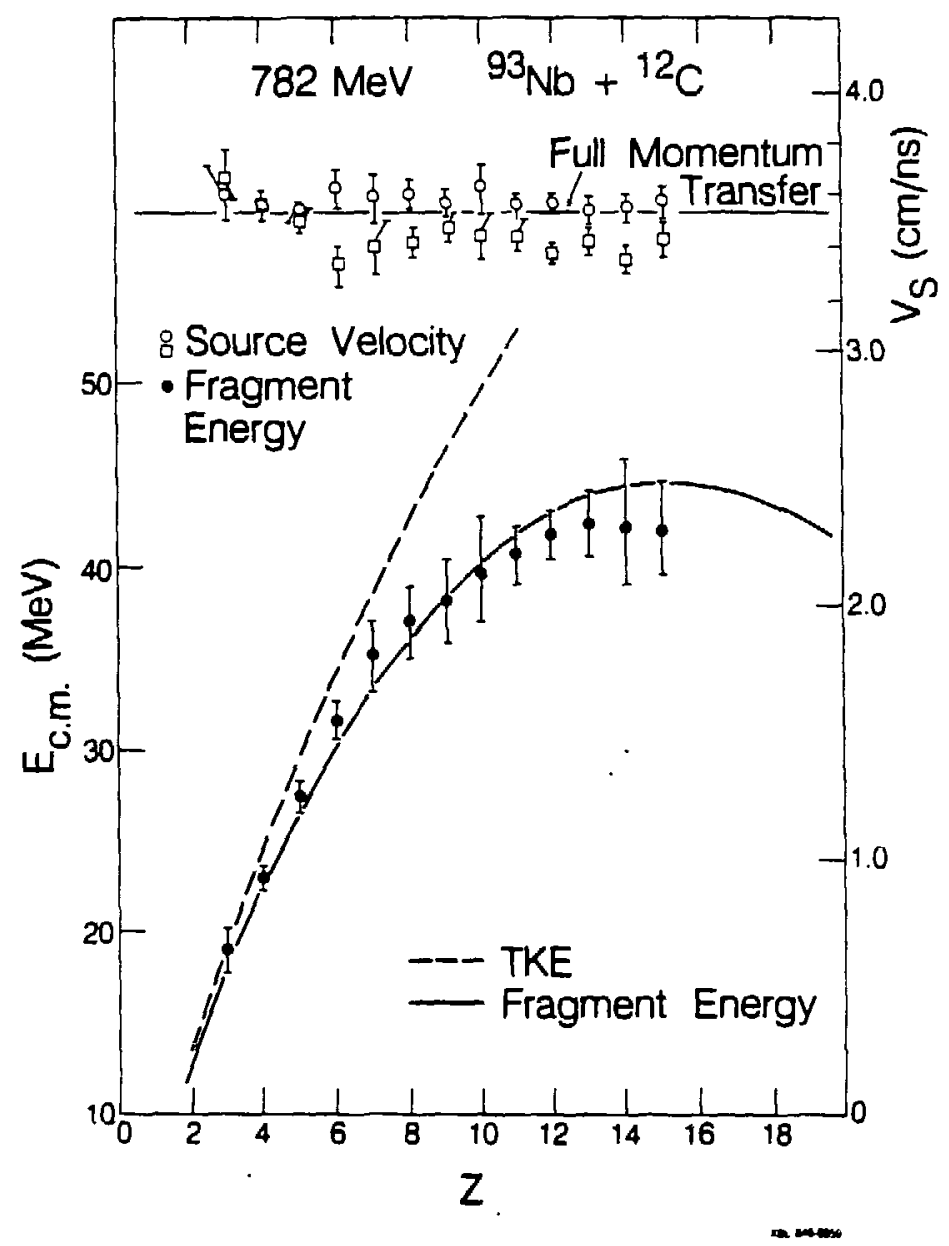

FIGURE 6 The deduced $c . m$. energies (filled cjrcles) and source velocities (open symbols) for the $93 \mathrm{Nb}+12 \mathrm{C}$ system. Source velocities were determined assuming that the product mass followed the line of B-stability (open circles) or the charge equilibration line (open squares). A Coulomb calculation for two spheres is shown both for the c.m. energy of the light fragment (solid line) and the total kinetic energy (dashed line). The value of the source velocity expected for full momentum transfer is indicated by the horizontal line.

function of charge asymmetry $\left(z_{\text {asy }}=z_{\text {detected }} / Z_{\text {total }}\right)$. The lack of enhancement in yield near the target $Z$ supports the compound nucleus origin of the products rather than a 
EMISSION: OF COMPLEX FRAGMENTS IN COMPOUND NUCLEUS DECAY

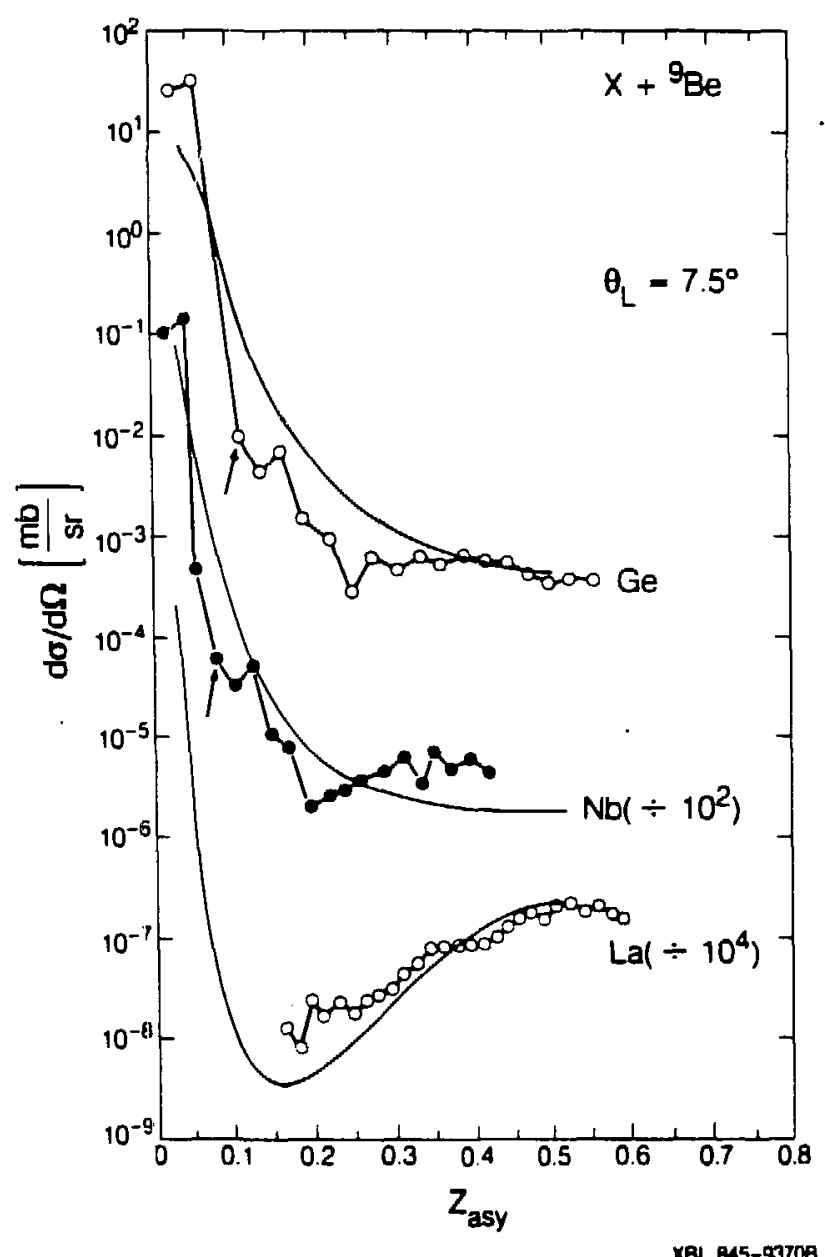

FIGURE 7 Center-of-mass cross sections for products from the ${ }^{74} \mathrm{Ge},{ }^{93} \mathrm{Nb}$ and $139_{\mathrm{La}}+9_{\mathrm{Be}}$ systems detected at $\theta_{\text {Lab }}=7.5^{\circ}$. The solid line is a liquid drop model calculation of the fragment yield at $\theta_{c}, \mathrm{~m}$. $=$ $30^{\circ}$. The arrows indicate the entrance channe $i$ as ymmetry. See text. Data below $\mathrm{z}_{\text {asy }}=0.15$ were not obtained for the La + Be system, due to a limited dynamic range of the telescope.

deep-inelastic origin. The yield from the ${ }^{74} \mathrm{Ge}+{ }^{9} \mathrm{Be}$ system, with a fissility parameter of $x=0.31$, decreases steadily as one moves towards symmetry. The yields from the ${ }^{93} \mathrm{Nb}+{ }^{9} \mathrm{Be}$ system $(x=0.40)$ are essentially constant from $Z_{\text {asy }}=0.2$ to 0.4 while the yields from the $139 \mathrm{La}+{ }^{9} \mathrm{Be}$ system $(x=0.50)$ show the characteristic fission peak at 


\section{L.G. MORETTO \\ $18 \mathrm{MeV} / \mathrm{UNb}+\mathrm{Al}$}

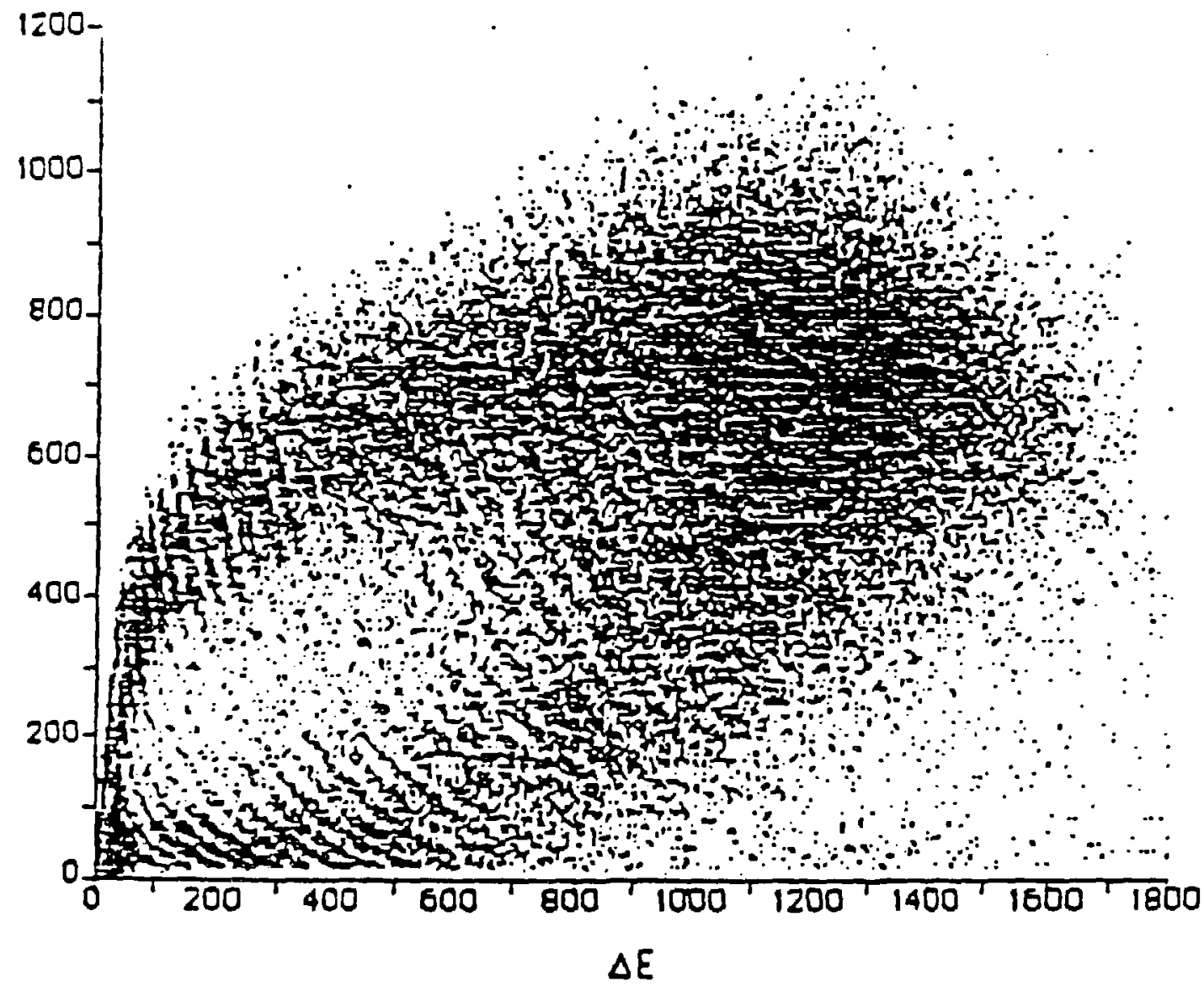

FIGURE 8 QE VS. E scatter plot for singles events produced in the reaction $18 \mathrm{MeV} / \mathrm{u} 93_{\mathrm{Nb}}+{ }^{9} \mathrm{Be}$. The detector subtended angles from $4^{\circ}$ to $12^{\circ}$. The two dark bands correspond to Coulomb emission from a compound nuc leus forward and backward in the center-of-mass.

symmetry. These three systems clearly exhibit the qualitative trends expected from the topological changes in the potential energy surface predicted by the liquid drop model (see Figure 1).

A quantitative comparison between these data and a compound nucleus calculation based upon the liquid drop model is also shown in Figure 7 . The agreement in absolute magnitude and in trend between this calculation and the data 
EMISSION OF COMPLEX FRAGMENTS IN COMPOUND NUCLEUS DECAY

confirms the compound nuclear origin of these fragments.

In summary, we have shown that fragments with atomic numbers covering the entire range of the mass asymmetry coordinate are produced from the decay of an excited compound nucleus. The observed $z$ distributions indicate that the topological transition expected at the Businaro-Gallone point does indeed take place in the region of $A \sim 100$. The exact position of the Businaro-Gallone point and its angular momentum dependence can in principle be established by a systematic study of the $Z$ or A distributions as the fissility parameter $x$ and the rotational parameter $y$ are varied.

Having established that complex fragments can be emitted by compound nuclei, and that at low energies they are only emitted by compound nuclei, the decision naturally came to see what is in fact going on at higher energies. Should any kind of compound nucleus be formed, it would decay abundantly by complex fragment emission due to its high excitation energy. This is the inescapable conclusion provided by statistical mechanics. More qualitatively, one could expect cross sections as large as several tens of millibarns per 2 . The corollary of this is that any additional "fancy" mechanism should ride on top of this already substantial compound cross section.

The reactions we chose are $\mathrm{Nb}+\mathrm{Be}, \mathrm{C}, \mathrm{Al}$ in reverse kinematics from 8.5 to $30-40 \mathrm{MeV} / \mathrm{u}$. As we shall see, the choice of a relatively light target simplifies the picture crucially because of the limitations in impact parameters and in the number of sources. While we explored first the upper part of the energy range at the Bevalac, 10 we shall begin with the lower energy data which we collected at GSI.11

Figure 8 demonstrates the advantages of reverse kinematics. In this picture we see the complex particles e'ents displayed in the E-AE plane. The remarkable cuble ridge is due to a simple kinematic effect. A single source is emitting fragments in the center of mass with energies independent of direction. Because of the large center-of-mass velocity, a given lab angle intersects the kinematic circle twice giving rise to a double solution. This simple observation aliows us to conclude that the process is binary, especially when for the measured velocities one obtains a Coulomb-like $Z$ dependence for the center-of-mass velocities. We also took coincidence data with another detector placed symetrically on the other side of the beam. These data confirm that the process is indeed binary, and that an upper solution fragment in one detector is in coincidence with a lower solution fragment 


\section{L.G. MORETTO}

in the other.

The pattern seen in Figure 8 evolves regularly and smoothly with bombarding energy and target. This indicates that indeed we are observing the same kinematic circle, boosted by different velocities of the center-of-mass which are in approximate agreement with those expected from complete fusion. Furthermore, reverse kinematics allows us to verify that what you see is all there is. When we go to wider angles we lose the intersection with the kinematic circle and we see nothing. So there are no other processes than the one we have described, and we can conclude that, up to $18.5 \mathrm{MeV} / \mathrm{u}$ "nihil sub sole novum, " nothing new under the sun but compound nucleus decay.

Proceeding to the better analyzed Bevalac data, we see more of the same. In Figure 9 the invariant cross sections plotted in the $Z-V$ plane show that the double solutions are retained up to $30 \mathrm{MeV} / \mathrm{u}$. Notice also that at very low Z's there is a trail of low velocity events which we call "big foot." This process is clearly target related, and may have to do with the onset of incomplete fusion. In this case the events are due to the target picking up a few nucleons for the projectile and a corresponding fraction of the momentum.

The velocities of the source clearly indicate a single source for all Z's with a velocity intermediate between the projectile velocity and the compound nucleus velocity but closer to the latter. The inferred incomplete momentum transfer in the direct kinematic solution is in good agreement with the standard momentum transfer systematics. Similarly the velocities in the center of mass are Coulomblike.

An example of charge distribution is shown in Figure 10 together with an absolute calculation. The ability to fit the absolute cross sections vs. Z with a compound nucleus model is in our eyes very significant, because it implies a statistical branching ratio between complex fragment emission and the dominant $n, p,{ }^{4} \mathrm{He}$ decay.

The coincidence data are shown in Figure 11 . The hatched bands are predicted on the bas is of the incomplete momentum transfer, of the resulting excitation energy, and of the sequential evaporation from the binary fragments calculated from the code PACE. 12 The overall picture is consistent with binary decay. However notice that in the case of the Al target at $30 \mathrm{MeV} / \mathrm{u}$ a number of events falls outside the expected band, indicating perhaps three or more body decay. A better appreciation of the coincidence data and of the calculations is given in Figure 12 where the 
EMISSION OF COMPLEX FRAGMENTS IN COMPOUND NUCLEUS DECAY

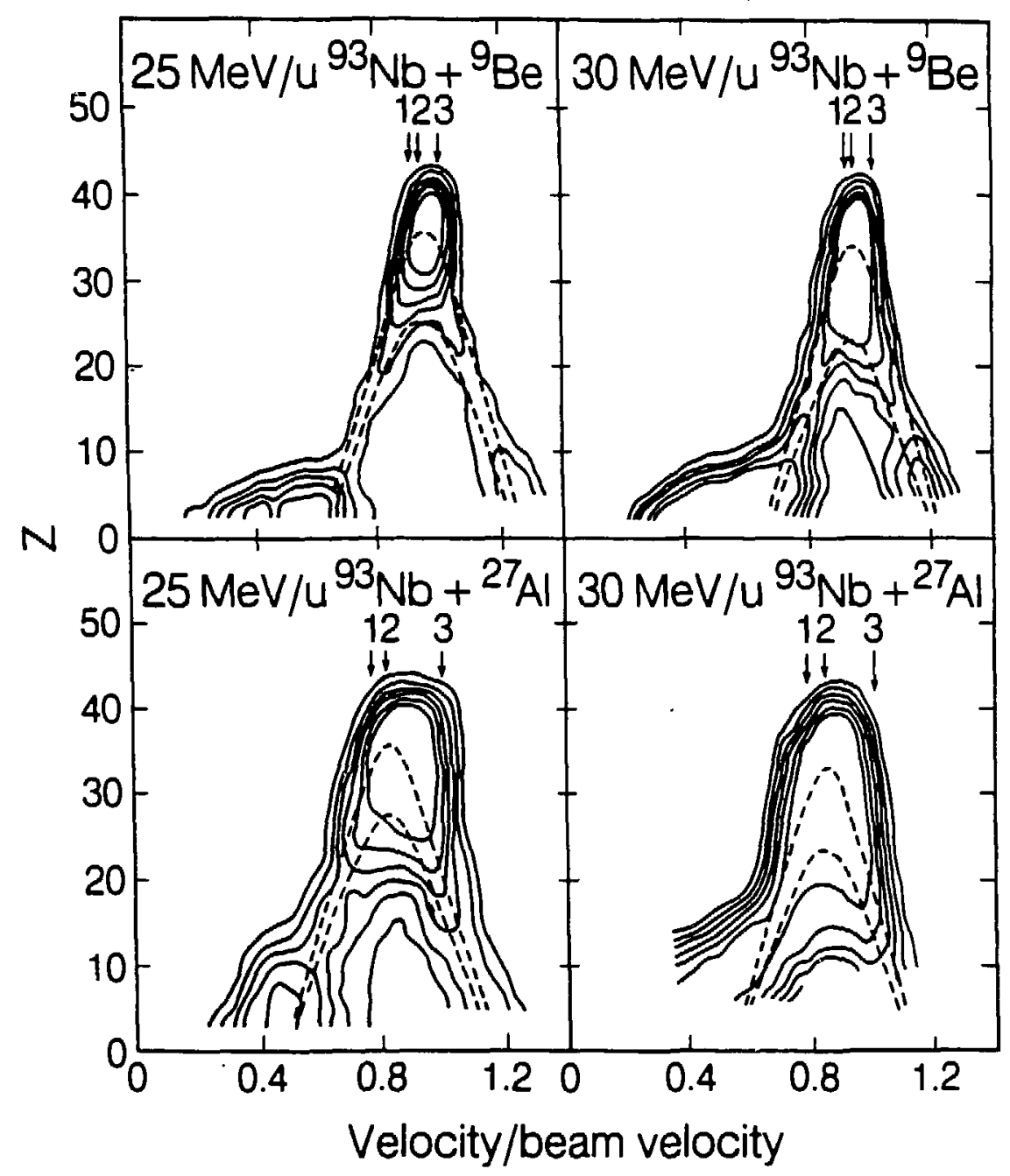

XBL 859-8978

FIGURE 9 Singles distribution of reaction products plotted as logarithmic contours of invariant cross section $\left[\left(1 / V^{2}\right)\left(\partial^{2} a / a(\Delta \partial V)\right]\right.$ in the Z-velocity plane. The arrows indicate the velocities for 1 ) full momentum transfer 2) the experimentally determined momentum transfer and 3 ) the beain. Calculated (dashed lines) average velocities of complex fragments for the maximum and minimum lab angles of the telescope $\left(3^{\circ}\right.$ and $\left.8^{\circ}\right)$ are indicated. 
L.G. MORETTO

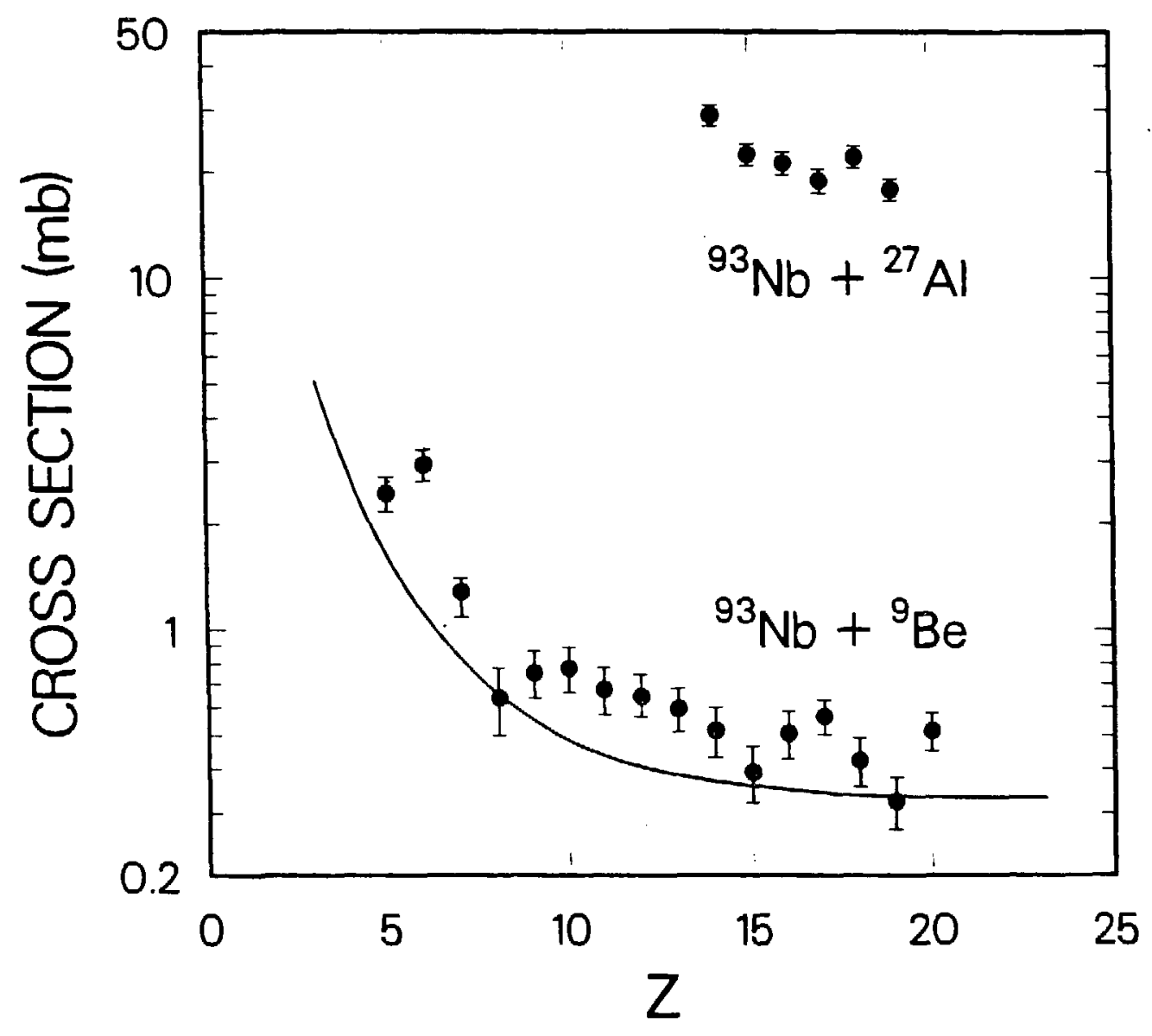

XBL 861-359

FIGURE 10 Angle-integrated cross sections (symbols) for complex fragments emitted from the reaction $30 \mathrm{MeV} / \mathrm{u}$ $93_{\mathrm{NB}}+27_{\mathrm{Al}}$ and ${ }^{9} \mathrm{Be}$. Liquid-drop model calculation (solid line) of the fragment yield for the latter system.

average sum of charges is plotted versus one of the charges. The dashed line is the primary sum inferred from the source velocity and the solid line is the calculation from PACE of 
EMISSION OF COMPLEX FRAGMENTS IN COMPOUND NUCLEUS DECAY

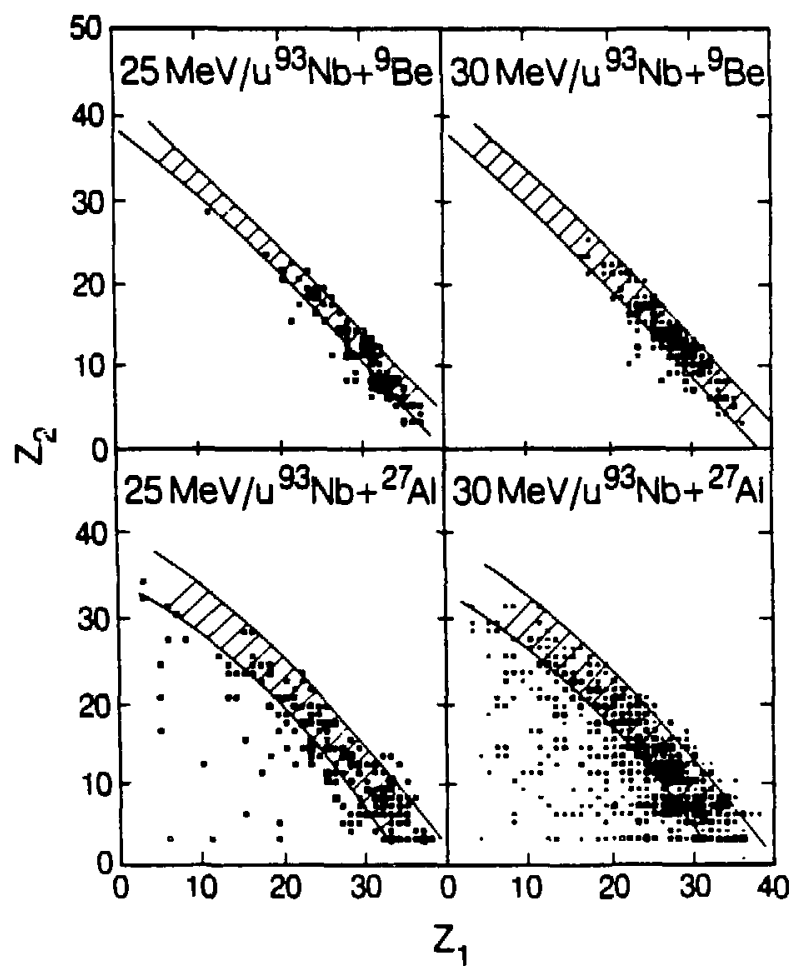

XBL 859-8976

FIGURE 11 Scatter plots of coincidence events between the $5.5^{\circ}$ telescope $\left(z_{1}\right)$ and the $-11^{\circ}$ telescope $\left(z_{2}\right)$.

The shaded areas represent an estimation of regions where binary events should lie following sequential evaporation from the primary fragments.

the sequential charge evaporation. The excellent agreement indicates a solid understanding of the incomplete fusion process, of the energy deposition, and of the binary decay followed by sequential evaporation.

What we can state with a good degree of confidence is that up to the highest explored energy, compound nucleus decay is by far the main source of complex fragments. An additional source at low A's is the "big foot" which is target related and seems to be consistent with the target picking up a few nucleons for the projectile and decaying in its turn. The very thorough exploration of angle and energy "phase space" allowed by reverse kinematics does not leave much room for any other processes. Can we conclude then that they are not there? I think we must wait for 
L.G. MORETTO

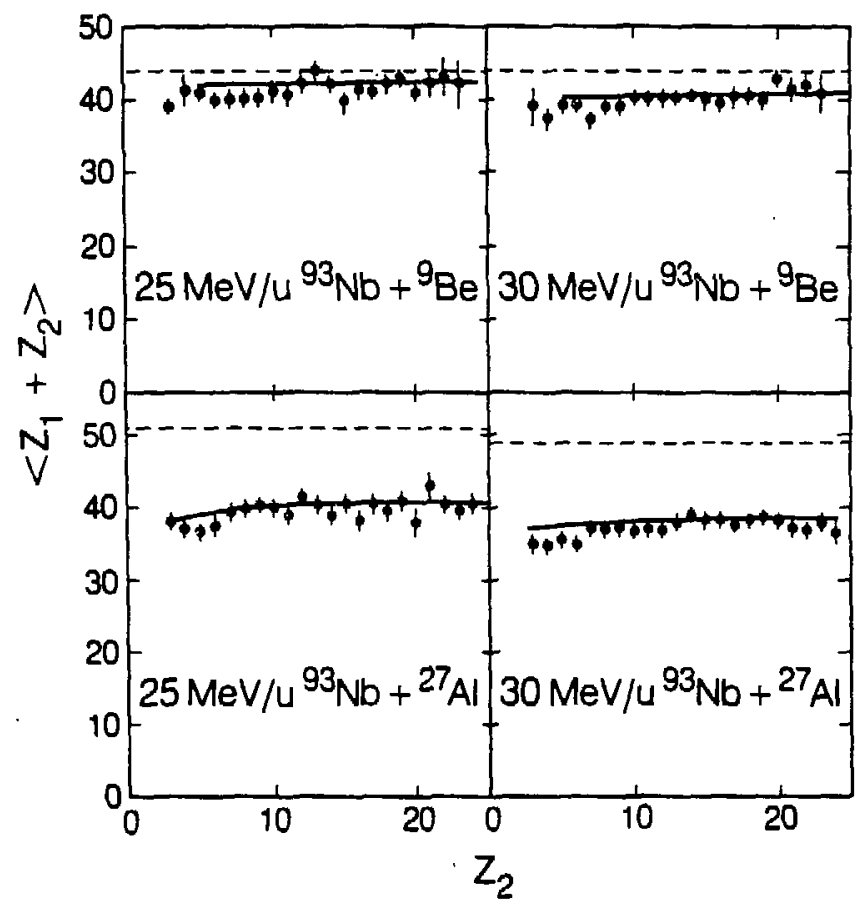

XEL $859-8977$

FIGURE 12 The mean sum, $\left\langle z_{1}+z_{2}\right\rangle$ of coincidence events (solid symbols) plotted as a function of $z_{2}$. The dashed lines indicate the average charge of the compound system as estimated from the mass transfer. The charge loss of binary events, due to sequential evaporation, was estimated using the PACE ${ }^{12}$ code and the residual $\left\langle z_{1}+z_{2}\right\rangle$ values are indicated by the solid curves.

ternary and quaternary events which will undoubtedly appear at higher energies. However even with these events one needs to be cautious. Ternary, quaternary and higher multiplicity events can originate from sequential binary decays. In fact, once one has a good excitation function for the binaries, it is a simple exercise to predict the rate of sequential ternaries and quaternaries. This will be the background, and it will not be small, on top of which we shall have to look in search of fancier mechanisms.

This work was supported by the Director, office of Energy Research, Division of Nuclear Physics of the Office of High Energy and Nuclear Physics of the U.S. Department of Energy under Contract DE-ACO3-76SF00098. 
EMISSION OF COMPLEX FRAGMENTS IN COMPOUND NUCLEUS DECAY

\section{REFERENCES}

1. E.K. Hyde, The Nuclear Properties of the Heavy Elements, Vol III: Fission Phenomena, Prentice-Hall, Inc., 1964, Ch. 11 and references there in

2. A.M. Poskanzer, G.W. Butler and E.K. Hyde, Phys. Rev. C3 (1971) 882; ibid C5 (1971) 1795.

3. J. Aichelin and J. Hüfner, Phys. Lett. 136B (1984) 15.

4. See for instance J.E. Finn, et al., Phys. Rev. Lett. $\underline{49}$ (1982) 1321.

5. L.G. Moretto, Nucl. Phys. A247 (1975) 211.

6. L.G. Sobotka, M.A. Padgett, G.J. Wozniak, G. Guarino, A.J. Pacheco, L.G. Moretto, Y. Chan, R.G. Stokstad, I. Tserruya, and S. Wald, Phys. Rev. Lett. 5l (1983) 2187.

7. M.A. McMahan et al., Phys. Rev. Lett. 54, 1995 (1985)

8. A.J. Sierk, Phys. Rev. Lett. $\underline{55}, 582$ (1985)

9. L.G. Sobotka, M.A. McMahan, R.J. McDonald, C. Signarbieux, G.J. Wozniak, M.L. Padgett, J.H. Gu, Z.H. Liu, Z.0. Yao and L.G. Moretto, Phys. Rev. Lett. 53, 2004 (1984)

10. R.J. Charity et al., Phys. Rev. Lett. 56, 1354 (1986)

11. R.J. Charity et al。, to be published

12. A. Gavron, Phys. Rev. C21, 230 (1980) 